\title{
Triage and optimization: A new paradigm in the treatment of massive pulmonary embolism
}

\author{
Chetan Pasrija, MD, ${ }^{\mathrm{a}}$ Aakash Shah, BS, ${ }^{\mathrm{a}}$ Praveen George, MD, ${ }^{\mathrm{b}}$ Anthony Kronfli, BS, ${ }^{\mathrm{a}}$

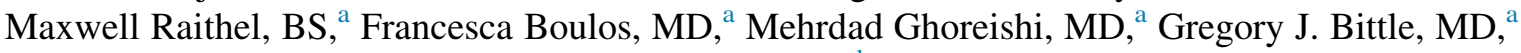 \\ Michael A. Mazzeffi, MD, ${ }^{\mathrm{c}}$ Lewis Rubinson, MD, PhD, ${ }^{\mathrm{d}}$ James S. Gammie, MD, ${ }^{\mathrm{a}}$ Bartley P. Griffith, MD, ${ }^{\mathrm{a}}$ \\ and Zachary N. Kon, MD
}

\begin{abstract}
Background: Massive pulmonary embolism (PE) remains a highly fatal condition. Although venoarterial extracorporeal membrane oxygenation (VA-ECMO) and surgical pulmonary embolectomy in the management of massive PE have been reported previously, the outcomes remain less than ideal. We hypothesized that the institution of a protocolized approach of triage and optimization using VA-ECMO would result in improved outcomes compared with historical surgical management.
\end{abstract}

Methods: All patients with a massive PE referred to the cardiac surgery service between 2010 and 2017 were retrospectively reviewed. Patients were stratified by treatment strategy: historical control versus the protocolized approach. In the historical control group, the primary intervention was surgical pulmonary embolectomy. In the protocol approach group, patients were treated based on an algorithmic approach using VA-ECMO. The primary outcome was 1-year survival.

Results: A total of 56 patients (control, $n=27$; protocol, $n=29$ ) were identified. All 27 patients in the historical control group underwent surgical pulmonary embolectomy, whereas 2 of 29 patients in the protocol approach group were deemed appropriate for direct surgical pulmonary embolectomy. The remaining 27 patients were placed on VA-ECMO. In the protocol approach group, 15 of 29 patients were treated with anticoagulation alone and 14 patients ultimately required surgical pulmonary embolectomy. One-year survival was significantly lower in the historical control group compared with the protocol approach group ( $73 \%$ vs $96 \% ; P=.02$ ), with no deaths occurring after surgical pulmonary embolectomy in the protocol approach group.

Conclusions: A protocolized strategy involving the aggressive institution of VAECMO appears to be an effective method to triage and optimize patients with massive PE to recovery or intervention. Implementation of this strategy rather than an aggressive surgical approach may reduce the mortality associated with massive PE. (J Thorac Cardiovasc Surg 2018;156:672-81)

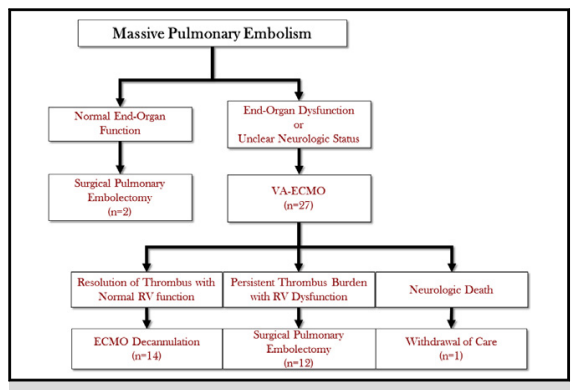

Algorithm for treatment of patients with massive pulmonary embolism.

\section{Central Message}

A protocolized approach to massive pulmonary embolism involving the use institution of venoarterial extracorporeal membrane oxygenation may reduce mortality compared with an aggressive surgical approach.

\section{Perspective}

A protocolized approach to massive pulmonary embolism, involving the aggressive institution of veno-arterial extracorporeal membrane oxygenation, appears to be an effective approach to triaging and optimizing patients to recovery or intervention. Compared with an aggressive surgical approach, implementation of this strategy may reduce the morbidity and mortality associated with massive pulmonary embolism.

See Editorial Commentaries pages 682 and 684.
From the ${ }^{\mathrm{a}}$ Division of Cardiac Surgery, Department of Surgery, ${ }^{\mathrm{b}}$ Department of Medicine, ${ }^{\mathrm{c}}$ Department of Anesthesiology, and ${ }^{\mathrm{d}}$ Shock Trauma Critical Care Unit, University of Maryland School of Medicine, Baltimore, Md; and ${ }^{\mathrm{e}}$ Department of Cardiothoracic Surgery, New York University Langone Health, New York, NY.

Read at the 97th Annual Meeting of The American Association for Thoracic Surgery, Boston, Massachusetts, April 29-May 3, 2017.

Received for publication May 4, 2017; revisions received Feb 16, 2018; accepted for publication Feb 25, 2018; available ahead of print May 2, 2018.

Address for reprints: Chetan Pasrija, MD, Division of Cardiac Surgery, University of Maryland, Baltimore, MD (E-mail: cpasrija@smail.umaryland.edu).

0022-5223/\$36.00

Copyright (c) 2018 by The American Association for Thoracic Surgery

https://doi.org/10.1016/j.jtcvs.2018.02.107
Within the spectrum of pulmonary embolism (PE), massive $\mathrm{PE}$, categorized primarily as systemic arterial hypotension secondary to right ventricular (RV) pressure overload,

Scanning this $Q R$ code will take you to a supplemental video. To view the AATS Annual Meeting Webcast, see the URL next to the webcast thumbnail. 


$$
\begin{array}{ll}
\text { Abbreviations and Acronyms } \\
\text { CT } & =\text { computed tomography } \\
\text { ICU } & =\text { intensive care unit } \\
\text { PE } & =\text { pulmonary embolism } \\
\text { RV } & =\text { right ventricular } \\
\text { TTE } & =\text { transthoracic echocardiography } \\
\text { VA-ECMO } & =\text { veno-arterial extracorporeal } \\
& \text { membrane oxygenation }
\end{array}
$$

remains substantially more fatal than submassive or simple PE. ${ }^{1,2}$ Treatment options for massive PE, including anticoagulation, systemic or catheter-based thrombolysis, and surgical pulmonary embolectomy, have all historically been associated with grave mortality rates between $15 \%$ and $80 \% .^{3-6}$ However, multiple recent series have demonstrated improved outcomes with aggressive and rapid surgical intervention for massive PE, with shortterm mortality rates between $6 \%$ and $15 \% .^{7-9}$

While our institution also established an aggressive surgical approach to massive PE, we found that the majority of mortalities were related to neurologic death associated with prolonged malperfusion and hypoxia before surgical intervention or to failure to recover end-organ and RV function in patients presenting with severe cardiogenic shock. Therefore, we established a highly protocolized approach to massive $\mathrm{PE}$, involving triage and optimization using veno-arterial extracorporeal membrane oxygenation (VAECMO). We hypothesized that the establishment of this protocol would result in improved outcomes compared with those associated with our historical surgical management.

\section{PATIENTS AND METHODS}

With Institutional Review Board approval, a retrospective review of the local Society of Thoracic Surgeons database was performed of all patients who underwent surgical pulmonary embolectomy or were placed on VAECMO for massive PE at a single institution between January 2010 and April 2017. A manual review of patient charts was then undertaken to confirm the diagnosis and obtain preoperative, perioperative, and postoperative variables and outcomes. PE was confirmed by computed tomography (CT) angiography in all cases. RV dysfunction was recorded based on preoperative and postoperative (before discharge) transthoracic echocardiography (TTE). Postoperative RV dysfunction (none, mild, moderate, or severe) was evaluated by an independent cardiologist, who both quantitatively and qualitatively assessed RV function to obtain an overall assessment of dysfunction.

Massive PE was defined, per the American Heart Association criteria, as systolic blood pressure $<90 \mathrm{~mm} \mathrm{Hg}$ for at least 15 minutes or the need for inotropic support, not due to a cause other than PE, or persistent profound bradycardia (heart rate $<40 \mathrm{bpm}$ with signs or symptoms of shock).

\section{Patients and Protocol}

Two groups were established: the protocol approach group and the historical control group. Patients in the protocol approach group (May 2015 to April 2017) strictly followed the algorithm shown in Figure 1. All patients with a massive PE who presented with normal end-organ function and intact neurologic exam were taken directly to the operating room for

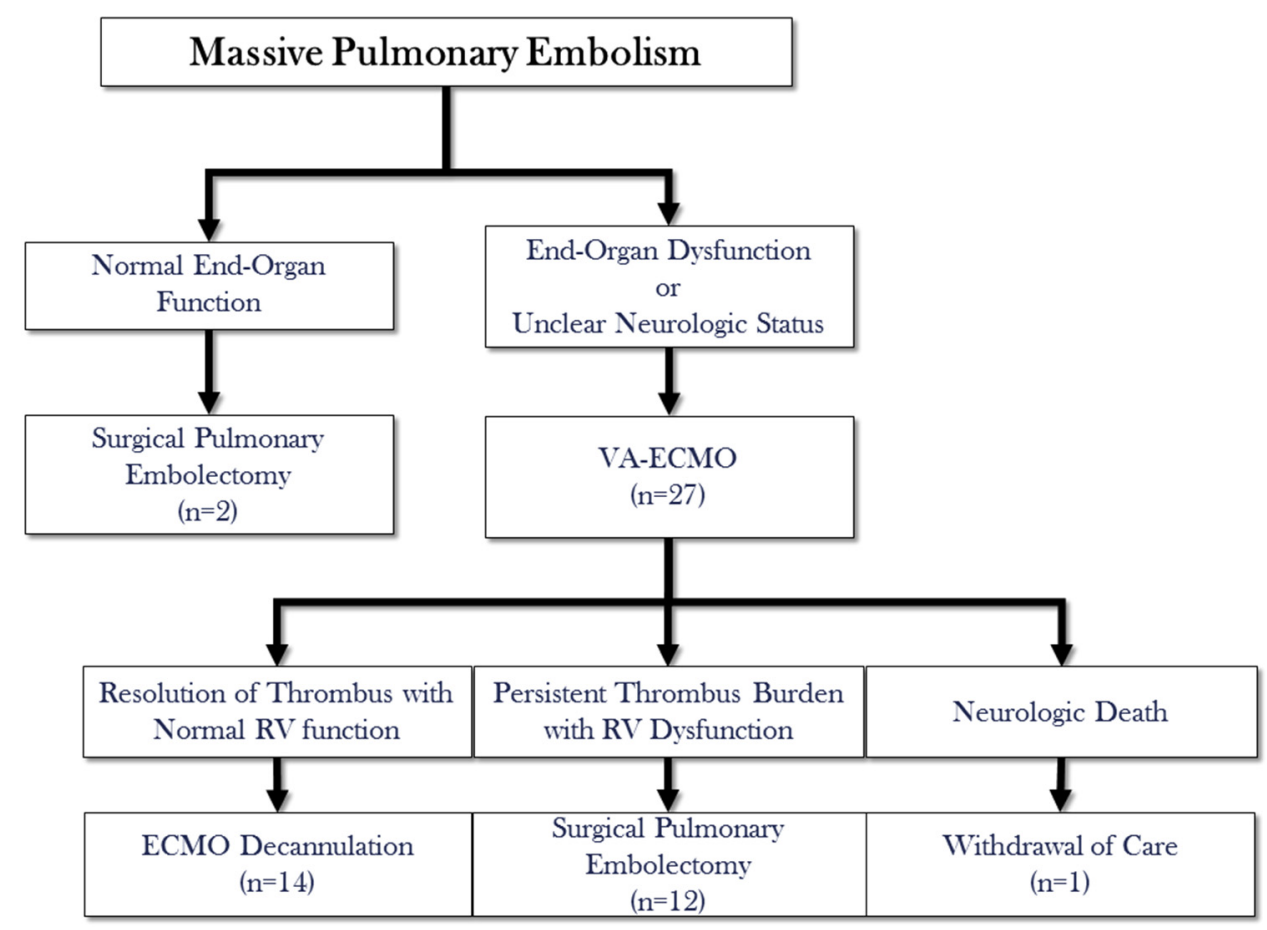

FIGURE 1. The algorithm for patients in the protocolized approach. VA-ECMO, Veno-arterial extracorporeal membrane oxygenation; $R V$, right ventricular; ECMO, extracorporeal membrane oxygenation. 
surgical pulmonary embolectomy. Any patient with a massive PE and endorgan dysfunction or an unclear neurologic exam was placed on VAECMO as the primary initial intervention.

VA-ECMO cannulation and management were provided as described previously. ${ }^{10}$ In brief, cannulation was performed via a venous drainage cannula placed in a femoral vein and an arterial return cannula placed in a femoral artery in the contralateral groin along with a distal perfusion cannula in the superficial femoral artery. ECMO flow was titrated up until the right ventricle was decompressed, and the patient was maintained on systemic anticoagulation with unfractionated heparin at a partial thromboplastin time of 72 to 113 seconds. ECMO support was continued until all of the following criteria were met: 1) neurologic status was determined if unclear before cannulation, 2) end-organ function was optimized, and 3) 3 to 7 days of heparin therapy were provided to allow for potential endogenous fibrinolysis. ECMO support was terminated early in patients with concerning findings, such as ECMO-related complications. In patients with neurologic death, organ donation was offered to the family and care was withdrawn.

In neurologically intact patients, repeat TTE was performed after all the forgoing criteria were met. In patients with normal RV size and function, ECMO decannulation was performed via a surgical cut-down in the operating room. In the subset of patients with continued RV dysfunction, surgical pulmonary embolectomy was performed, as described previously. ${ }^{11}$ In brief, all patients were placed on cardiopulmonary bypass with mild hypothermia. Central aortic and bicaval venous cannulation was performed. Separate incisions were made into the right and left main pulmonary arteries, and the thrombus was removed in its entirety up to the subsegmental level. The operation was routinely performed on a beating heart without placement of an aortic cross-clamp, unless required for a concomitant procedure.

The primary planned intervention for all patients in the historical control group was surgical pulmonary embolectomy. Use and management of VA-ECMO was at the discretion of the surgeon. Surgical technique and postoperative management were as described previously. ${ }^{11}$ The arbitrary placement of a patient on VA-ECMO at the discretion of the treating surgeon who did not follow the algorithmic strategy did not qualify for stratification to the protocol approach group. Patients who did not undergo surgical pulmonary embolectomy or who were not placed on VA-ECMO as part of the described algorithm were excluded from this study $(n=3)$.

\section{Clinical Outcomes}

The primary study outcome was 1-year survival. Secondary outcomes included in-hospital survival, postembolectomy survival, time from cardiac arrest to intervention, peri-intervention cardiac arrest, postoperative ECMO support, mechanical ventilation duration, intensive care unit (ICU) length of stay, hospital length of stay, need for tracheostomy, acute kidney injury as defined by the Society of Thoracic Surgeons, new hemodialysis at discharge, sepsis, postoperative cerebrovascular complication, RV dysfunction at discharge, and ECMO-related complications, including bleeding requiring blood product transfusion on ECMO and vascular complications requiring intervention while supported on ECMO. ECMO duration was defined as the time from cannulation to operative intervention (ECMO decannulation or surgical pulmonary embolectomy). Periintervention arrest was defined as cardiac arrest occurring during ECMO cannulation or induction of anesthesia.

\section{Statistical Analysis}

All data analyses were performed with JMP 11.0 software (SAS Institute, Cary, NC). Continuous variables are presented as median with interquartile range (IQR). Skewed continuous variables were compared using the Wilcoxon rank-sum test, and normally distributed variables were compared using the Student $t$ test. Categorical variables are presented as number and percentage and compared using the Fisher exact test. A $P$ value of $<.05$ was considered statistically significant. Postoperative proportions of RV dysfunction were compared using the $\chi^{2}$ test. Survival was calculated as the time from initial intervention to death. Patients were censored at the last known follow-up or, in those still alive at the end of the study, using the Kaplan-Meier method. No patients were censored secondary to missing survival data. Differences in survival curves were assessed using the log-rank test. Predictors of survival were assessed using a univariate Cox regression model and are presented as hazard ratio with $95 \%$ confidence interval.

\section{RESULTS}

\section{Patient Demographic Data and Clinical Presentation}

A total of 56 patients, including 29 patients in the protocol approach group and 27 in the historical control group, were identified with a median age of 55 years (IQR, 4464 years). Risk factors for PE were comparable between the 2 groups, including deep vein thrombosis $(72 \%)$, obesity $(57 \%)$, immobility $(53 \%)$, and recent surgery $(41 \%)$ (Table 1). All patients in this study had an elevated troponin level (median, $0.9 \mathrm{ng} / \mathrm{mL}$; IQR, 0.3-1.8 ng/mL) with severe RV dysfunction and a RV-to-left ventricular (LV) ratio $>1.0$ based on TTE, similar in the 2 groups. Cardiopulmonary resuscitation before consultation was required in $21 \%$ of the patients overall $(21 \%$ in the protocol approach group and $22 \%$ in the historical control group; $P=$ not significant). Consultation occurred during active cardiopulmonary resuscitation in 2 patients in the protocol approach group, and both patients were placed on VAECMO while receiving active cardiopulmonary resuscitation. No patients in the historical control group were placed on VA-ECMO or taken to the operating room during active cardiopulmonary resuscitation. Systemic thrombolysis was provided before consultation in $18 \%$ of the patients $(21 \%$ in the protocol approach group and $15 \%$ in the historical control group; $P=\mathrm{NS}$ ) (Table 2).

\section{Initial Management and Outcomes}

Twenty-seven of 29 patients $(93 \%)$ in the protocol approach group and 6 of $27(22 \%)$ in the historical control group were placed on VA-ECMO as an initial intervention $(P<.001)$ (Figure 2). In the protocol approach group, all patients were placed on VA-ECMO as the primary planned intervention, with a median duration of 5.8 days. In the historical control group, patients were placed on VA-ECMO as a rapid bridge to the primary planned intervention of surgical pulmonary embolectomy, with a median duration of 1.0 days $(P<.001)$. This resulted in a median time from consultation to surgery of 79 hours longer in the protocol approach group. Although there was a similar incidence of preconsultation cardiac arrest, the time from arrest to intervention was significantly shorter in the protocol approach group $(P=.05)$. Furthermore, there was a $15 \%$ incidence of intraoperative arrest during the induction of anesthesia in the historical control group, compared with no pericannulation or intraoperative arrests in the protocol approach group $(P=.05)$. Postoperative ECMO support, 
TABLE 1. Patient demographic characteristics and risk factors for PE

\begin{tabular}{|c|c|c|c|}
\hline Characteristic & Historical control group $(n=27)$ & Protocol approach group $(n=29)$ & $P$ value \\
\hline Age, y, median (IQR) & $60(45-67)$ & $50(41-59)$ & .11 \\
\hline Male sex, $n(\%)$ & $15(56)$ & $15(52)$ & .80 \\
\hline BMI, $\mathrm{kg} / \mathrm{m}^{2}$, median (IQR) & $33(26-37)$ & $30(27-37)$ & .53 \\
\hline \multicolumn{4}{|l|}{ Race, n (\%) } \\
\hline African-American & $7(26)$ & $13(45)$ & .17 \\
\hline Caucasian & $17(63)$ & $14(48)$ & .30 \\
\hline Other & $3(11)$ & $2(7)$ & .66 \\
\hline \multicolumn{4}{|l|}{ Risk factors for PE, n (\%) } \\
\hline Deep vein thrombosis & $21(78)$ & $18(62)$ & .25 \\
\hline Immobility & $14(52)$ & $16(55)$ & 1 \\
\hline Malignancy & $4(15)$ & $2(7)$ & .41 \\
\hline Obesity & $18(67)$ & $14(48)$ & .19 \\
\hline Recent surgery $(<1 \mathrm{mo})$ & $12(44)$ & $11(38)$ & .79 \\
\hline \multicolumn{4}{|l|}{ Comorbid conditions, $\mathrm{n}(\%)$} \\
\hline Coronary artery disease & $0(0)$ & $0(0)$ & 1 \\
\hline Congestive heart failure & $0(0)$ & $0(0)$ & 1 \\
\hline Chronic obstructive pulmonary disease & $2(7)$ & $2(7)$ & 1 \\
\hline Cardiovascular disease & $0(0)$ & $0(0)$ & 1 \\
\hline Diabetes & $8(30)$ & $6(21)$ & .54 \\
\hline Dyslipidemia & $7(26)$ & $6(21)$ & .76 \\
\hline Hypertension & $15(56)$ & $14(48)$ & .61 \\
\hline Renal failure (CKD stage 3 or worse) & $1(4)$ & $2(7)$ & 1 \\
\hline Renal failure requiring hemodialysis & $1(4)$ & $1(3)$ & 1 \\
\hline
\end{tabular}

$I Q R$, Interquartile range; $B M I$, body mass index; $P E$, pulmonary embolism; $C K D$, chronic kidney disease

secondary to persistent RV dysfunction, was required in 1 patient in the protocol approach group and in 4 patients in the historical control group.
Surgical pulmonary embolectomy was performed in $100 \%$ of patients in the historical control group and $48 \%$ of those in the protocol approach group. In the protocol

TABLE 2. Preintervention variables

\begin{tabular}{|c|c|c|c|}
\hline Variable & Historical control group $(n=27)$ & Protocol approach group $(\mathrm{n}=29)$ & $P$ value \\
\hline \multicolumn{4}{|l|}{ Preconsultation } \\
\hline Cardiac arrest, $\mathrm{n}(\%)$ & $6(22)$ & $6(21)$ & 1 \\
\hline Cardiac arrest to intervention, $\mathrm{h}$, median (IQR) & $12(9-15)$ & $5(4-7)$ & .05 \\
\hline Systemic thrombolysis, $\mathrm{n}(\%)$ & $4(15)$ & $6(21)$ & .73 \\
\hline Consultation to intervention, $\mathrm{h}$, median (IQR) & $4(3-13)$ & $1(1-3)$ & $<.001$ \\
\hline Uncertain neurologic status at consultation, $\mathrm{n}(\%)$ & $13(48)$ & $13(45)$ & 1 \\
\hline \multicolumn{4}{|l|}{ RV strain } \\
\hline Troponin, ng/mL, median (IQR) & $0.37(0.13-0.64)$ & $1.35(0.51-2.22)$ & $<.001$ \\
\hline Severe RV dysfunction, $\mathrm{n}(\%)$ & $27(100)$ & $29(100)$ & 1 \\
\hline $\mathrm{RV} / \mathrm{LV}>1.0, \mathrm{n}(\%)$ & $27(100)$ & $29(100)$ & 1 \\
\hline \multicolumn{4}{|l|}{ Hemodynamics } \\
\hline Heart rate, bpm, median (IQR) & $104(85-123)$ & $124(105-134)$ & .03 \\
\hline Systolic blood pressure, mm Hg, median (IQR) & $113(96-121)$ & $98(83-122)$ & .20 \\
\hline Use of inotropes/vasopressors, $\mathrm{n}(\%)$ & $18(67)$ & $19(66)$ & .78 \\
\hline \multicolumn{4}{|l|}{ Respiratory status } \\
\hline Respiratory rate, breaths/min, median (IQR) & $28(26-33)$ & $26(20-32.25)$ & .50 \\
\hline $\mathrm{PaO}_{2} / \mathrm{FiO}_{2}$, median (IQR) & $113(93-185)$ & $103(83-250)$ & .84 \\
\hline $\mathrm{FiO}_{2}$, median (IQR) & $0.9(0.4-1.0)$ & $1.0(0.4-1.0)$ & .29 \\
\hline Intubated, n (\%) & $19(70.4)$ & $15(51.7)$ & .18 \\
\hline
\end{tabular}

$I Q R$, Interquartile range; $R \mathrm{~V}$, right ventricular; $\mathrm{LV}$, left ventricular; $\mathrm{PaO}_{2}$, partial pressure of arterial oxygen; $\mathrm{FiO}_{2}$, fraction of inspired oxygen. 


\section{Protocol Approach}

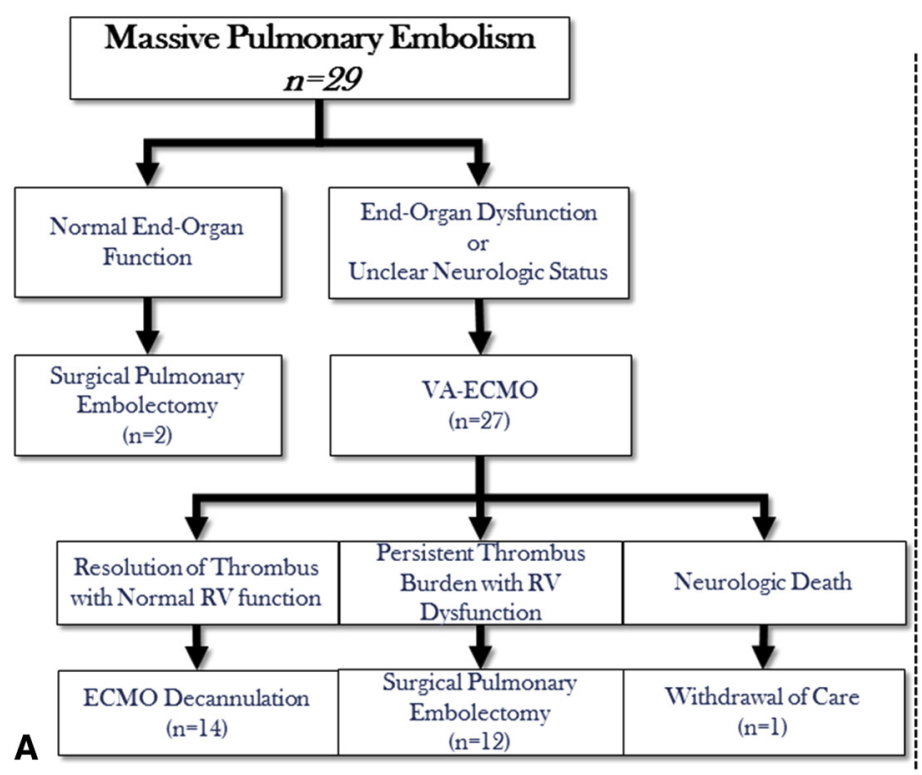

Historical Control

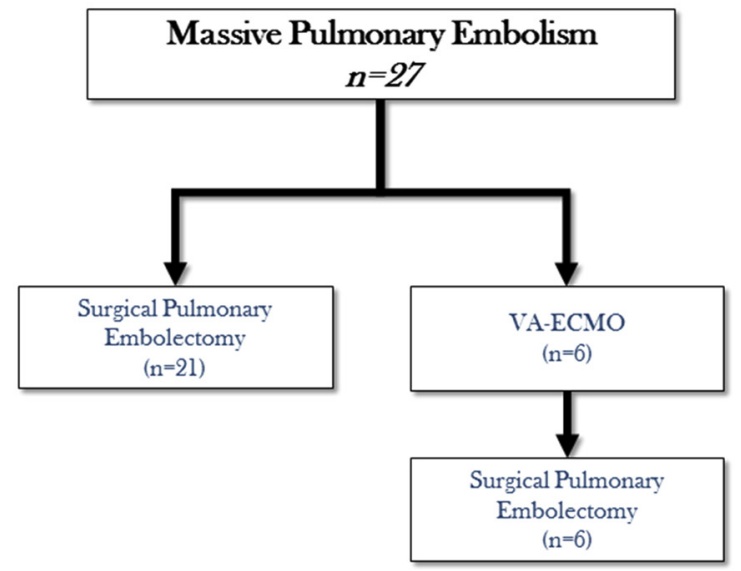

B

FIGURE 2. Therapeutic strategy for patients in the protocol approach group (A) and historical control group (B). VA-ECMO, Veno-arterial extracorporeal membrane oxygenation; $R V$, right ventricular; $E C M O$, extracorporeal membrane oxygenation.

approach group, 2 patients were deemed appropriate for direct surgical pulmonary embolectomy according to the algorithm. Twelve other patients underwent surgical pulmonary embolectomy at a median of 3.7 days (IQR, 2.55.2 days) following ECMO cannulation after demonstrating residual RV dysfunction with pulmonary hypertension on repeat TTE and persistent thrombus on CT angiography. The remaining 15 patients in the protocol approach group were treated with anticoagulation alone after repeat TTE demonstrated normal RV function, with decannulation performed at a median of 6.0 days (IQR, 5.7-7.1 days) after ECMO cannulation (Table 3).

\section{Posttherapy Outcomes and Survival}

The duration of mechanical ventilation was similar in the 2 groups, but ICU length of stay and hospital length of stay were significantly longer in the protocol approach group. In a subset analysis of survivors, these increases were no longer statistically significant. Acute kidney injury after therapeutic intervention (ECMO cannulation or surgical

TABLE 3. ECMO and operative characteristics

\begin{tabular}{|c|c|c|c|}
\hline Characteristic & Historical control group $(\mathbf{n}=27)$ & Protocol approach group $(\mathbf{n}=29)$ & $P$ value \\
\hline \multicolumn{4}{|l|}{ ECMO } \\
\hline Preoperative ECMO support, $\mathrm{n}(\%)$ & $6(22)$ & $27(93)$ & $<.001$ \\
\hline ECMO duration, d, median (IQR) & $1.0(0.3-1.7)$ & $5.8(4.3-6.7)$ & $<.001$ \\
\hline \multicolumn{4}{|l|}{ Operative } \\
\hline Operative intervention, $\mathrm{n}(\%)$ & $27(100)$ & $14(48)$ & $<.001$ \\
\hline Consultation to surgery, $\mathrm{h}$, median (IQR) & $5(3-19)$ & $84(22-120)$ & $<.001$ \\
\hline Peri-intervention arrest, $\mathrm{n}(\%)$ & $4(15)$ & $0(0)$ & .05 \\
\hline Operative time, min, median (IQR) & $186(157-233)$ & $210(198-242)$ & .06 \\
\hline CPB time, min, median (IQR) & $74(52-112)$ & $78(62-95)$ & .65 \\
\hline Cross-clamp, n (\%) & $8(30)$ & $2(14)$ & .45 \\
\hline Myocardial ischemia time, min, median (IQR) & $45(38-54)$ & $17(17-18)$ & .15 \\
\hline \multicolumn{4}{|l|}{ Therapeutic intervention } \\
\hline Anticoagulation alone, $\mathrm{n}(\%)$ & $0(0)$ & $15(52)$ & $<.001$ \\
\hline Surgical pulmonary embolectomy, n (\%) & 27 (100) & $14(48)$ & $<.001$ \\
\hline
\end{tabular}

$E C M O$, Extracorporeal membrane oxygenation; $I Q R$, interquartile range; $C P B$, cardiopulmonary bypass time. 
TABLE 4. Outcomes

\begin{tabular}{|c|c|c|c|}
\hline Outcome & Historical control group $(n=27)$ & Protocol approach group $(n=29)$ & $P$ value \\
\hline \multicolumn{4}{|l|}{ ECMO support complications } \\
\hline Bleeding, $\mathrm{n} / \mathrm{N}(\%)$ & $3 / 6(50)$ & $4 / 27(15)$ & .09 \\
\hline Vascular complication, $\mathrm{n} / \mathrm{N}(\%)$ & $0 / 6(0)$ & $2 / 27(7)$ & 1 \\
\hline Postoperative ECMO support, $\mathrm{n} / \mathrm{N}(\%)$ & $4 / 27(15)$ & $1 / 14(7)$ & .64 \\
\hline Mechanical ventilation duration, d, median (IQR) & $3.3(0.8-10.5)$ & $1.6(0.1-7.0)$ & .43 \\
\hline ICU LOS, d, median (IQR) & $5.6(3.4-12.5)$ & $10.1(7.6-16.4)$ & .05 \\
\hline Hospital LOS, d, median (IQR) & $8.0(5.2-15.5)$ & $12.1(10.0-19.5)$ & .03 \\
\hline Tracheostomy, n (\%) & $3(11)$ & $2(7)$ & .66 \\
\hline Acute kidney injury, n (\%) & $4(15)$ & $4(14)$ & 1 \\
\hline New dialysis (at discharge), $\mathrm{n}(\%)$ & $1(4)$ & $0(0)$ & .48 \\
\hline New CVA, n (\%) & $0(0)$ & $0(0)$ & 1 \\
\hline Sepsis, n $(\%)$ & $1(4)$ & $1(3)$ & 1 \\
\hline Postoperative vascular complication, n (\%) & $2(7)$ & $2(7)$ & 1 \\
\hline \multicolumn{4}{|l|}{ RV dysfunction (predischarge), n/N (\%) } \\
\hline None & $12 / 16(75)$ & $23 / 25(92)$ & .13 \\
\hline Mild & $4 / 16(25)$ & $2 / 25(8)$ & \\
\hline Moderate & $0 / 16(0)$ & $0 / 25(0)$ & \\
\hline Severe & $0 / 16(0)$ & $0 / 25(0)$ & \\
\hline In-hospital survival postembolectomy, $\mathrm{n} / \mathrm{N}(\%)$ & $22 / 27(82)$ & $14 / 14(100)$ & .15 \\
\hline Overall in-hospital survival, $\mathrm{n}(\%)$ & $22(82)$ & $28(97)$ & .10 \\
\hline One-year survival, $\%$ & 73 & 96 & .02 \\
\hline
\end{tabular}

$E C M O$, Extracorporeal membrane oxygenation; $I Q R$, interquartile range; $I C U$, intensive care unit; $L O S$, length of stay; $C V A$, cerebrovascular accident; $R V$, right ventricular.

pulmonary embolectomy) and a new dialysis requirement at discharge was similar in the 2 groups. There was a nonstatistically significant trend toward less RV dysfunction in the protocol approach group (Table 4).

In-hospital survival was $97 \%$ in the protocol approach group and $82 \%$ in the historical control group $(P=.10)$, with postembolectomy in-hospital survival of $100 \%$ versus $82 \%(P=.15)$. The 1 -year Kaplan-Meier estimated survival was $96 \%$ in the protocol approach group and $73 \%$ in the historical control group $(P=.02)$ (Figure 3). Overall median follow-up was 525 days. Three patients who were alive at the last known follow-up were censored before

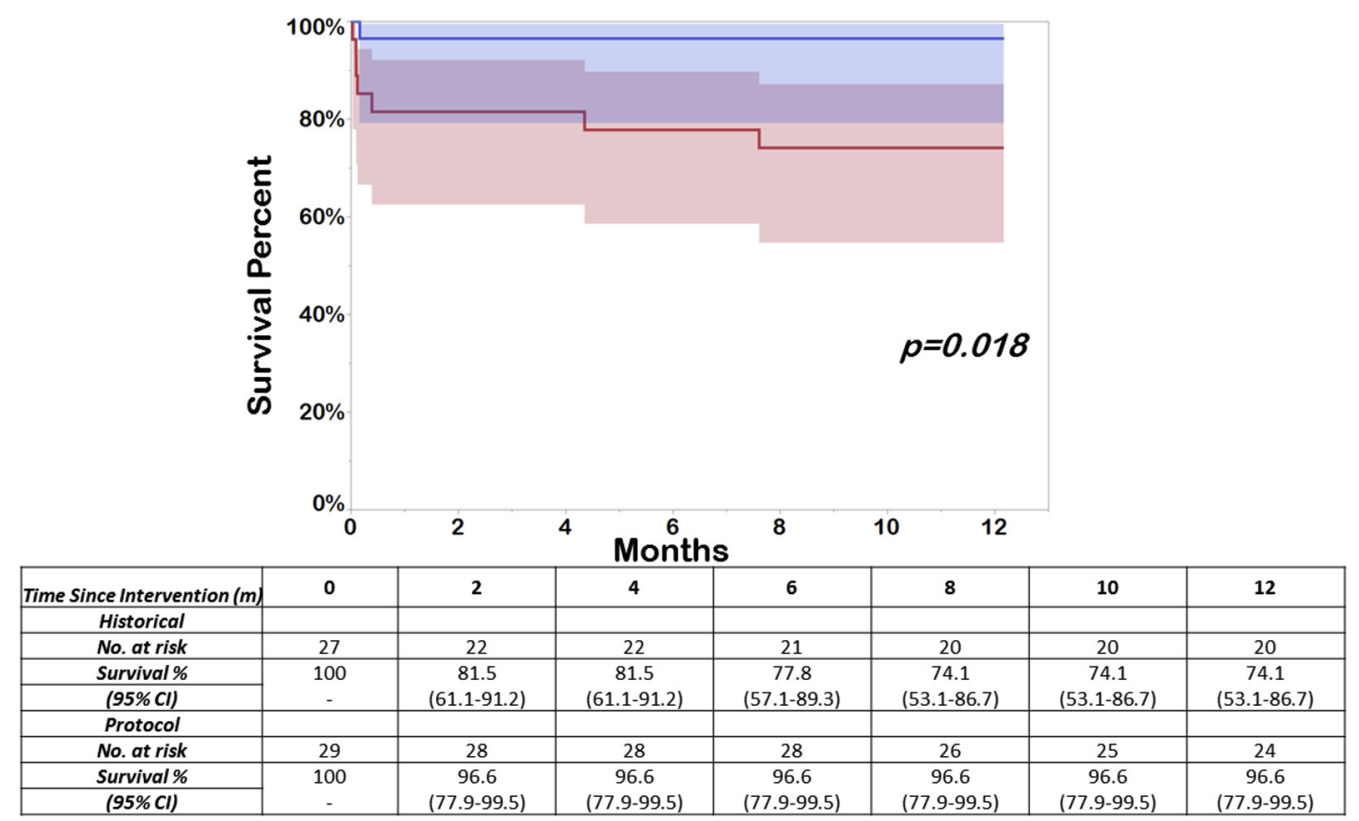

FIGURE 3. Kaplan-Meier survival curves for patients in the protocol approach group (blue) and historical control group (red). Tick marks signify censored patients. $C I$, Confidence interval. 
TABLE 5. Univariate Cox regression model for mortality

\begin{tabular}{|c|c|c|c|}
\hline Variable & HR & $95 \%$ CI & $P$ value \\
\hline Age (per year) & 1.021 & 0.976-1.069 & .36 \\
\hline BMI (per 1) & 1.032 & $0.966-1.104$ & .35 \\
\hline Troponin (per 1) & 0.316 & $0.067-1.498$ & .15 \\
\hline Heart rate (per 10 bpm) & 0.932 & $0.698-1.245$ & .63 \\
\hline $\begin{array}{l}\text { Systolic blood pressure (per } \\
\qquad 10 \mathrm{~mm} \mathrm{Hg} \text { ) }\end{array}$ & 0.938 & $0.691-1.275$ & .68 \\
\hline Respiratory rate (per 1 bpm) & 0.985 & $0.901-1.078$ & .75 \\
\hline $\mathrm{PaO}_{2} / \mathrm{FiO}_{2}($ per $10 \mathrm{~mm} \mathrm{Hg}$ ) & 0.970 & $0.857-1.098$ & .63 \\
\hline Intubated & 0.197 & $0.024-1.605$ & .13 \\
\hline Cardiac arrest & 0.833 & $0.168-4.130$ & .82 \\
\hline $\begin{array}{l}\text { Uncertain neurologic status at } \\
\text { presentation }\end{array}$ & 0.331 & $0.064-1.706$ & .19 \\
\hline $\begin{array}{l}\text { Consultation to surgery time (per } \\
\text { day) }\end{array}$ & 0.591 & $0.255-1.371$ & .22 \\
\hline ECMO to surgery time (per hour) & 0.567 & $0.336-0.958$ & .04 \\
\hline $\begin{array}{l}\text { Historical approach (compared } \\
\text { with protocol approach) }\end{array}$ & 8.350 & $1.027-67.894$ & .01 \\
\hline
\end{tabular}

1 year. Moreover, Cox regression analysis identified the historical control group as a predictor for mortality, with a hazard ratio of 8.350 (95\% confidence interval, 1.027-67.894; $P=.01)$ (Table 5). Within the historical control group, 4 of 5 in-hospital mortalities were patients with an uncertain neurologic status who were taken urgently to the operating

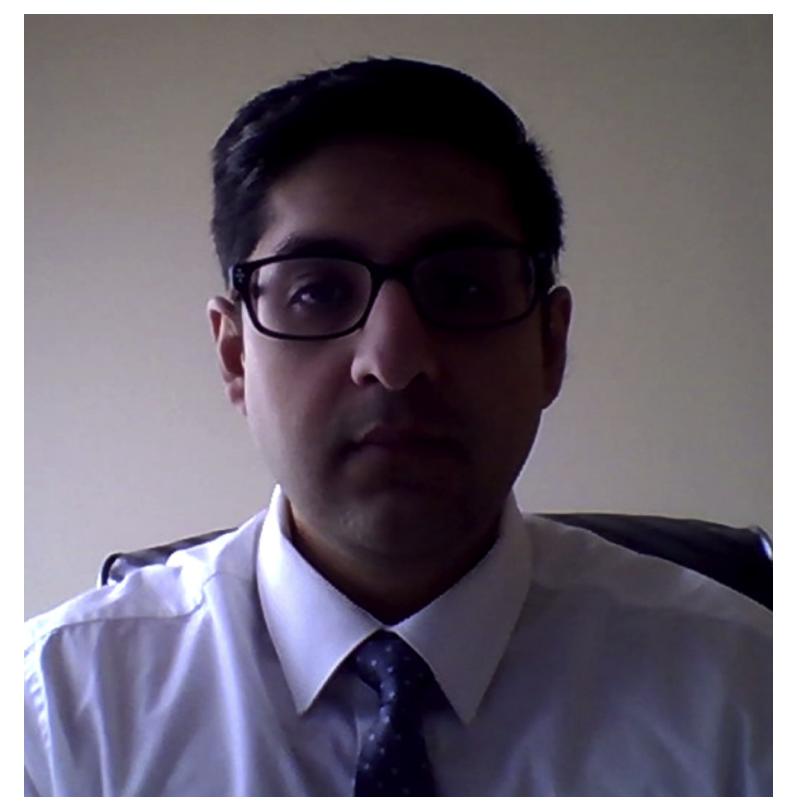

VIDEO 1. The lead author discusses the manuscript and the importance of this research. Video available at: https://www.jtcvs.org/article/S00225223(18)30931-0/fulltext. room due to their hemodynamic extremis but failed to experience neurologic recovery. One in-hospital mortality was secondary to multisystem organ failure and diffuse intravascular coagulation after surgical pulmonary embolectomy. Among the 6 patients who were placed on VA-ECMO preoperatively in the historical control group, there was a $50 \%$ (3 of 6) in-hospital and 1-year mortality. There were 2 postdischarge mortalities in the historical control group, in an 85 -year-old female who had a prolonged surgical course including mediastinitis and multiple intubations and a 63year-old female who required postoperative VA-ECMO, tracheostomy, and extended time in a rehabilitation center.

In the protocol approach group, the 1 mortality was a patient who required $>60$ minutes of cardiopulmonary resuscitation before consultation and experienced neurologic death.

\section{DISCUSSION}

This study demonstrates that a protocolized approach involving the aggressive institution of VA-ECMO may reduce mortality compared with an early, aggressive surgical approach for massive PE. Furthermore, VA-ECMO appears to be an effective support strategy to triage and optimize patients with massive PE to recovery or further intervention (Video 1).

While performing a high-volume of surgical pulmonary embolectomies over the past 7 years, we found that patients were commonly transferred to the operating room with signs of malperfusion or in frank cardiogenic shock. As such, there was a high rate $(15 \%)$ of cardiac arrest after induction of anesthesia. Furthermore, multiple patients required high-dose inotropes and vasopressors, or VAECMO, postoperatively secondary to persistent RV stunning after weaning from cardiopulmonary bypass. This often led to a prolonged postoperative course and increased mortality. Therefore, we developed an aggressive protocolized strategy to place patients with massive PE with evidence of end-organ dysfunction on VA-ECMO as an initial intervention to allow for recovery of hemodynamics and end-organ function. Importantly, we also found that RV function normalized in $52 \%$ of the patients (15 of 29) treated based on the algorithm with anticoagulation alone, reducing the overall need for aggressive surgical intervention.

Patients with a massive PE in this series frequently presented with an uncertain neurologic status secondary to cardiac arrest, hypoperfusion, hypoxia, and/or paralysis with intubation. Given their urgent clinical status, patients in the historical control group were often taken to the operating room with uncertainty regarding neurologic status or potential for recovery. Even in patients who did undergo preoperative imaging, early CT scans were often inconclusive. Four of 13 patients in this group who presented with an unclear neurologic status did not regain neurologic 
function, and after a prolonged course in the hospital, care was withdrawn.

The institution of VA-ECMO, along with restoring perfusion, allows for early declaration of neurologic function; neurologic status was determined within 48 hours in all patients in the protocol approach group. In the 1 patient in the protocol approach group who experienced neurologic death, a rapid diagnosis was made, and organ donation was offered to the family. Interestingly, despite a similar rate of preconsultation cardiac arrests, there was only 1 neurologic death in the protocol approach group, compared with 4 neurologic deaths in the historical control group. We postulate that this difference may be the result of earlier restoration of perfusion in the protocol approach group, with significant less time from cardiac arrest to intervention (12 hours vs 5 hours).

This study does suggest that the aggressive implementation of VA-ECMO may prolong ICU and hospital lengths of stay. In the protocol approach group, $44 \%$ of the patients (12 of 27) placed on VA-ECMO required surgical pulmonary embolectomy for persistent RV dysfunction, possibly prolonging their hospital course. However, the differences in length of stay were no longer significant in the analysis of survivors. In addition, this may be an inherent trade-off for overall improved survival and neurologic outcome. Moreover, we routinely perform awake VA-ECMO cannulation and attempt extubation in intubated patients with massive PE on VA-ECMO to limit mechanical ventilation time and debilitation, with $>75 \%$ of patients on VAECMO in the protocol approach group engaging in physical therapy, including walking, while awaiting decannulation or surgical intervention. This perhaps is distinct from an intubated prolonged postoperative ICU length of stay.

Although other series have described VA-ECMO for PE, in-hospital mortality in these studies ranged from $20 \%$ to $60 \% .^{12-15}$ These patients were commonly placed on ECMO as a salvage intervention rather than a primary intervention, often after failure of other therapies. Moreover, once on ECMO, no apparent algorithmic approach was applied. In addition, the time from ECMO to further therapeutic intervention was variable, perhaps not allowing recovery of end-organ function or determination of neurologic status. In this study, the early implementation of VA-ECMO in the protocol approach group may have prevented cardiac arrest and prolonged malperfusion. Meanwhile, the extended time on VA-ECMO allowed recovery of end-organ and RV function, leading to improved overall and postembolectomy survival.

\section{Limitations}

This study is a retrospective cohort comparison, with the associated inherent limitations. Although the 2 patient groups were similar in baseline characteristics, there may be unmeasured differences. Furthermore, the protocolized approach was only performed between 2015 and 2017, whereas patients in the historical control group ranged from 2010 to 2016. Undetected differences in operative approach or ICU management might have affected outcomes. Furthermore, other confounders due to the 6-year time period of the historical control group may be present. In addition, we do not have a medical control arm for comparing our therapeutic outcomes. Although registry data suggest a high mortality in this subset of patients, a prospective study comparing the protocolized approach and standard therapy is needed to better elucidate and confirm these differences and outcomes. This study was performed at an experienced VA-ECMO center, in which complications related to cannulation and management have been minimized. We do believe that this approach remains an excellent strategy for less experienced ECMO centers, but VA-ECMO cannulation and management must be approached with the same meticulous attention to detail as in other cardiac surgical operations.

\section{CONCLUSIONS}

A protocolized approach involving the aggressive institution of VA-ECMO appears to be an effective method to triage and optimize patients with massive PE to recovery or intervention. Implementation of this strategy rather than an early, aggressive surgical approach appears to reduce the morbidity and mortality associated with massive PE.

\section{Webcast}

You can watch a Webcast of this AATS meeting presentation by going to: https://aats.blob.core.windows.net/ media/17AM/2017-05-03/RM302-304/05-03-17_Room302304_0730_Pasrija.mp4.

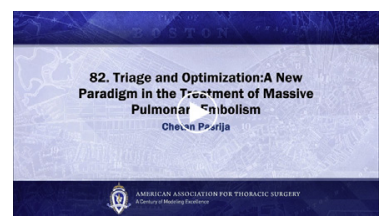

\section{Conflict of Interest Statement}

Authors have nothing to disclose with regard to commercial support.

\section{References}

1. Kasper W, Konstantinides S, Geibel A, Olschewski M, Heinrich F, Grosser KD,
et al. Management strategies and determinants of outcome in acute major pulmo-
nary embolism: results of a multicenter registry. J Am Coll Cardiol. 1997;30:
1165-71.
2. Goldhaber SZ, Visani L, De Rosa M. Acute pulmonary embolism: clinical out-
comes in the international cooperative pulmonary embolism registry (ICOPER).
Lancet. 1999;353:1386-9.
3. Kucher N, Rossi E, De Rosa M, Goldhaber SZ. Massive pulmonary embolism.
Circulation. 2006;113:577-82. 
4. Riera-Mestre A, Jiménez D, Muriel A, Lobo JL, Moores L, Yusen RD, et al. Thrombolytic therapy and outcome of patients with an acute symptomatic pulmonary embolism. J Thromb Haemost. 2012;10:751-9.

5. Lin BW, Schreiber DH, Liu G, Briese B, Hiestand B, Slattery D, et al. Therapy and outcomes in massive pulmonary embolism from the emergency medicine pulmonary embolism in the real world registry. Am J Emerg Med. 2012;30: 1774-81.

6. Smith SB, Geske JB, Kathuria P, Cuttica M, Schimmel DR, Courtney DM, et al. Analysis of national trends in admissions for pulmonary embolism. Chest. 2016; 150:35-45.

7. Neely RC, Byrne JG, Gosev I, Cohn LH, Javed Q, Rawn JD, et al. Surgical embolectomy for acute massive and submassive pulmonary embolism in a series of 115 patients. Ann Thorac Surg. 2015;100:1245-51; discussion 1251-2.

8. Keeling WB, Leshnower BG, Lasajanak Y, Binongo J, Guyton RA, Halkos ME, et al. Midterm benefits of surgical pulmonary embolectomy for acute pulmonary embolus on right ventricular function. J Thorac Cardiovasc Surg. 2016;152: $872-8$

9. Leacche M, Unic D, Goldhaber SZ, Rawn JD, Aranki SF, Couper GS, et al. Modern surgical treatment of massive pulmonary embolism: results in 47 consecutive patients after rapid diagnosis and aggressive surgical approach. J Thorac Cardiovasc Surg. 2005; 129:1018-23.

10. Pasrija C, Kronfli A, George P, Raithel M, Boulos F, Herr DL, et al. Utilization of veno-arterial extracorporeal membrane oxygenation for massive pulmonary embolism. Ann Thorac Surg. 2018;105:498-504.

11. Pasrija C, Kronfli A, Rouse M, Raithel M, Bittle GJ, Pousatis S, et al. Outcomes after surgical pulmonary embolectomy for acute submassive and massive pulmonary embolism: a single-center experience. J Thorac Cardiovasc Surg. 2018;155: 1095-106.e2.

12. Dolmatova EV, Moazzami K, Cocke TP, Elmann E, Vaidya P, Ng AF, et al. Extracorporeal membrane oxygenation in massive pulmonary embolism. Heart Lung. 2017;46:106-9.

13. Maggio P, Hemmila M, Haft J, Bartlett R. Extracorporeal life support for massive pulmonary embolism. J Trauma. 2007;62:570-6.

14. Hashiba K, Okuda J, Maejima N, Iwahashi N, Tsukahara K, Tahara Y, et al. Percutaneous cardiopulmonary support in pulmonary embolism with cardiac arrest. Resuscitation. 2012;83:183-7.

15. Corsi F, Lebreton G, Bréchot N, Hekimian G, Nieszkowska A, Trouillet JL, et al. Life-threatening massive pulmonary embolism rescued by venoarterialextracorporeal membrane oxygenation. Crit Care. 2017;21:76.

Key Words: massive pulmonary embolism, veno-arterial extracorporeal membrane oxygenation, surgical pulmonary embolectomy

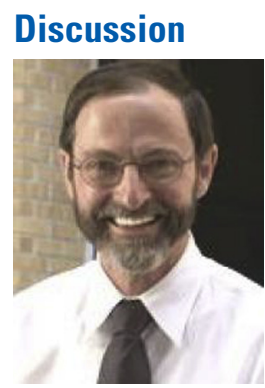

Dr Lyle Joyce (Milwaukee, Wis). I would like to thank the authors for giving me the opportunity to discuss their manuscript at this Centennial Meeting of the AATS. Dr Pasrija, thank you for providing me with the full manuscript to review before your presentation.

The authors are to be commended for their superb outcomes in dealing with this highly fatal condition of massive pulmonary embolism. They presented data to highlight 2 important issues in dealing with this deadly disease: first, the use of a protocolized strategy and, second, the importance of early aggressive intervention of the surgical treatment now that we have appropriate mechanical support devices, in this case ECMO, available. They have illustrated these points by comparing the outcomes in treating this problem in their practice over 2 time periods: the early period, which was more of the pre-ECMO era, and then their more recent experience with the use of ECMO, which has become highly successful in treating cardiorespiratory shock. Dr Pasrija, I have a few observations and then some questions for you.

First, you stated that all patients that did not receive a surgical pulmonary embolectomy and were not entered into the protocolized approach were excluded from the study. Why were they not put in a pre-protocolized group of patients? Were these patients that survived without surgical treatment, and had they been protocolized, would they have received ECMO and then fall into that group of survivors who did not require surgical excision? The issue that I am questioning here is whether these really are comparable groups of patients. Are there survivors in the early group who just didn't get included because they didn't get ECMO?

Second, you have nicely shown that the right ventricular function normalized in 52\% of your patients who were placed on early ECMO with anticoagulation alone and therefore did not need surgical embolectomy. This once again demonstrates how resilient the right heart can be if just unloaded for a period of time. However, in cases where oxygenation is not the problem, this does raise the question of whether we should be using temporary RVADs rather than ECMO, which would reduce the risk of possible left-sided complications such as strokes and peripheral emboli problems. What are your thoughts about that?

Third, in your description of the technique of pulmonary embolectomy, you state that each PA is opened and the thrombus is extracted down to the level of the subsegmental branches. If the clot does not extract well, do you stop there or do you have other tricks that you use, such as retrograde flushing of the pulmonary veins or massaging the lungs and things like that?

Fourth, you stated that patients are placed on peripheral ECMO by bilateral groin cannulation, and yet you described extubating them and getting them up and walking them around. How do you manage that?

Once again, I want to congratulate you on a very nice study. As you have stated, there are limitations with a retrospective study, but you have documented the value of setting up protocols, following them, analyzing the outcomes, and only then modifying the protocol as needed to improve your outcomes even more. There are many approaches that can be taken to treat this problem, but one thing is clear: with the technology that we have today, there is no excuse for not immediately aggressively treating patients with massive pulmonary emboli.

Thank you. 


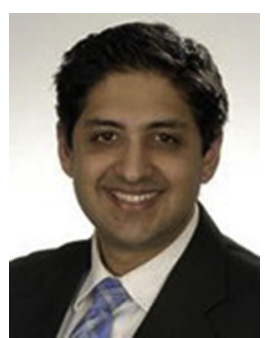

Dr Chetan Pasrija (Baltimore, Md). Thank you, Dr Joyce. To address your questions in the order that you asked them: first, for our exclusion criteria for this paper, we excluded all patients who didn't follow the protocol, essentially. For instance, we had a few patients who were placed on VA-ECMO as a salvage intervention after they arrested getting catheter-based intervention or TPA. We considered that different from our protocolized approach, which is placing the patient on VA-ECMO and preventing those issues of cardiac arrest from potentially other therapies.

With regard to your second question, using a temporary RVAD versus VA-ECMO, we found that peripheral VAECMO can be done in awake patients and generally fairly easily at the bedside. In our experience, hypoxia has never been the driving issue behind putting these patients on ECMO, but rather right ventricular failure, and we think that peripheral VA-ECMO at the bedside provides a quick and easy approach to getting these patients to the ICU and getting them extubated and moving around.

Regarding your third question, if we find that the thrombus is particularly stuck, we actually found that in about $15 \%$ of the patients there was more acute and chronic disease, and in those patients we do have to perform partial pulmonary thromboendarterectomy. Part of the benefit of putting these patients on VA-ECMO is that you don't take these patients to the operating room in the middle of the night. You can have an organized team, you can have somebody who does these operations, does PTEs, and is able to perform that operation, if necessary, at the time of the operation in an elective fashion.

Regarding your fourth question, as we have gotten more comfortable with VA-ECMO, we found that often times these patients are fairly comfortable with minimal sedation on ECMO, and once you get them extubated, as long as you protect your cannula as well, we are able to get them out of bed and walking around with minimal complications.

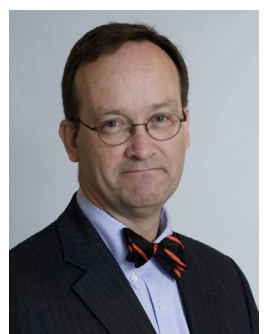

Dr Thoralf Sundt (Boston, Mass). Great paper. I am delighted to see you present these kinds of data, and I agree with Lyle that this is a disease that we used to just sort of shrug and say, well, they died of a massive PE, but that's not true anymore.

It's also worth pausing for a moment to remember that this is why Dr Gibbon developed the heart-lung machine. He was a research fellow with Dr Churchill when he sat at the bedside of a woman after a cholecystectomy who died of a pulmonary embolus and dedicated his life to building the heart-lung machine for exactly what has happened here. Unfortunately for the
MGH, Dr Churchill thought the effort was going nowhere, and that's why the best of the work happened at Jefferson.

I learned this approach to PE-support to treat the right ventricular dysfunction and pulmonary vasoconstriction using ECMO-from Lyle some years ago.

So my question is when to pull the trigger on medical therapy versus surgical therapy or even catheter-based therapy. We have established a pulmonary embolism response team where there is a GoTo meeting/telephone call with folks from vascular medicine, emergency medicine, hematology, cardiology, et cetera, to discuss the case and determine which direction we are going to go. There are many other options besides the one presented here. How do you decide for a given patient with a PE that comes in the front door, or through the ED, whether they go the surgical route, a catheter-based route, or a medical route?

Thank you.

Dr Pasrija. Thanks, Dr Sundt. Just to echo your first point, the first talk I ever went to was a Fitzgibbon Lecture that Dr Bartlett gave, and it was about a PE patient who was placed on VA-ECMO, and that patient was 30 years old and came to that lecture.

Regarding your question, how do we manage the different therapeutic options, in sudden massive PE patients, we have right now actually a prospective trial comparing catheter-based therapy to surgical intervention, and we have been more aggressive about establishing a similar team with a critical care physician, a vascular medicine physician, vascular surgery, and cardiothoracic surgery. However, in the massive PE population, we found that taking those patients directly either to surgery, catheter-based intervention, or medical intervention has a high risk of problematic aspects, such as cardiac arrest or failure of that therapy.

So in the massive PE population, our strategy is now if they have end-organ dysfunction or an unclear neurologic status, they go on VA-ECMO. And as Dr Joyce pointed out, more than half of those patients never need a surgical intervention. They can just be decannulated and leave the hospital with normal right ventricular function.

Dr Sundt. Thanks. A pulmonary embolism response team is a great innovation, though.

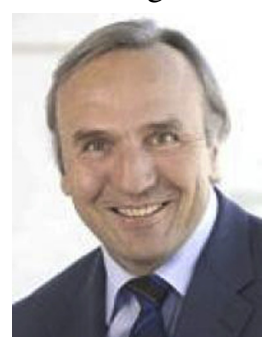

Dr Friedrich Mohr (Leipzig, Germany). I just want to draw your attention to a paper of Professor Starck from Berlin who uses VA-ECMO and a steerable AngioVac system and extracts the thrombus from the pulmonary artery. I think this is a very elegant way in some cases. I'm not saying in all cases. Just for your information. Maybe you can include that in your paper as well. 